\title{
Analytical solutions for a two-level system driven by a class of chirped pulses
}

\author{
Pankaj K. Jha ${ }^{1, *}$ and Yuri V. Rostovtsev ${ }^{1,2}$ \\ ${ }^{1}$ Institute for Quantum Science and Engineering and Department of Physics, Texas A\&M University, College Station, Texas 77843, USA \\ ${ }^{2}$ Department of Physics, University of North Texas, 1155 Union Circle 311427, Denton, Texas 76203, USA
}

(Received 14 April 2010; published 6 July 2010)

\begin{abstract}
We present analytical solutions for the problem of a two-level atom driven by a class of chirped pulses. The solutions are given in terms of Heun functions. By use of the appropriate chirping parameters, an enhancement of four orders of magnitude in the population transfer is obtained.
\end{abstract}

DOI: 10.1103/PhysRevA.82.015801

PACS number(s): 42.50.-p

Interaction of coherent optical pulses with quantum systems is a fundamental problem [1] that is closely related to important applications [2,3]. Nowadays, laser systems produce controlled, intense, ultrashort optical pulses [4]. Various technologies have been used for pulse shaping [5], which allows researchers to provide coherent optical control [6] of excitation in quantum systems; this has a broad range of applications from nonlinear laser spectroscopy to generation of coherent radiation. For example, chirped pulses [2,5] are used to produce maximal coherence in atomic and molecular systems. Maximal coherence can be used for generation of short-wavelength radiation, which has been a focus of research recently in an atomic system under the action of a far-off-resonance strong pulse of laser radiation; it has been shown that such pulses can excite remarkable coherence in high-frequency far-detuned transitions; and this coherence can be used for efficient generation of soft x-ray and ultraviolet radiation [3,7,8]. Maximal coherence can also be used for molecular spectroscopy, for example, time-resolved coherent Raman spectroscopy, to obtain molecule-specific signals from molecules that can serve as marker molecules for bacterial spores [2].

In this Brief Report, we investigate two classes of chirped pulses for which the problem can be solved exactly in analytical form. By use of the appropriate chirping parameters, the population transfer, after the the pulse is gone, can be optimized, and for the pulse considered here an enhancement by four orders of magnitude was obtained. An unchirped pulse corresponding to the Heun and confluent Heun equations has recently been investigated extensively in Ref. [8] where we included an estimate of the energy of emission of soft x-ray and ultraviolet radiation via excited quantum coherence in the atomic system. The estimate shows good potential for a source of coherent radiation based on the discussed mechanism.

The equation of motion for the probability amplitudes for the states $|a\rangle$ and $|b\rangle$ [see Fig. 1(a)] of a two-level atom $[9,10]$ interacting with a classical field (under the rotatingwave approximation) with nonzero chirping [12] is given as

$$
\begin{gathered}
\dot{C}_{a}=i \Omega(t) e^{i \vartheta(t)} C_{b}, \\
\dot{C}_{b}=i \Omega^{*}(t) e^{-i \vartheta(t)} C_{a},
\end{gathered}
$$

\footnotetext{
*pkjha@physics.tamu.edu
}

where $\vartheta(t)=\Delta t+\phi(t)$. Here $\Delta=\omega-v[11]$ and $\Omega(t)=$ $\wp \mathcal{E}(t) / 2 \hbar$. Let us define the dimensionless parameters as

$$
\tau=\alpha t, \quad \beta=\frac{\Delta}{\alpha}, \quad \gamma=\frac{\Omega_{0}}{\alpha} .
$$

To solve for $C_{a}$, we can get a second-order linear differential equation for $C_{a}$ from Eq. (1), which in terms of the dimensionless parameters of Eq. (2) is given as

$$
\ddot{C}_{a}-\left(i \beta+\frac{\dot{\Omega}(\tau)}{\Omega(\tau)}+i \dot{\phi}(\tau)\right) \dot{C}_{a}+\Omega^{2}(\tau) C_{a}=0 .
$$

\section{A. Class I: Heun equation}

To find an analytical solution for Eq. (3), we introduce a new variable $\varphi=\varphi(\tau)$ defined by

$$
\tau=(1 / 2) \ln \left[\varphi^{\mu} /(1-\varphi)^{\mu+\lambda}\right]
$$

and make an ansatz for the pulse envelope $\Omega(\tau)$ and the chirping function $\phi(\tau)$ as

$$
\begin{gathered}
\Omega(\tau)=\left(\frac{2 \varphi(1-\varphi)}{(c-\varphi)}\right)^{1 / 2}\left(\frac{\gamma}{\mu+\lambda \varphi}\right), \\
\dot{\phi}(\tau)=\left(\frac{-2 c \zeta+2[(\zeta+\xi)+c(\zeta+\eta)] \varphi}{(\varphi-c)(\mu+\lambda \varphi)}\right) .
\end{gathered}
$$

In terms of the variable $\varphi(\tau)$ and the definition of $\Omega(\tau)$ and $\dot{\phi}(\tau)$ from Eq. (5), Eq. (3) takes the form

$C_{a}^{\prime \prime}+\left(\frac{\rho}{\varphi}+\frac{\sigma}{\varphi-1}+\frac{v}{\varphi-c}\right) C_{a}^{\prime}+\frac{a b \varphi-q}{\varphi(\varphi-1)(\varphi-c)} C_{a}=0$,

where $c>1$ and

$$
\begin{gathered}
\rho=\frac{1}{2}-i\left(\zeta+\frac{\beta \mu}{2}\right), \quad \sigma=\frac{1}{2}+i\left(\frac{\beta(\mu+\lambda)}{2}-\eta\right) \\
v=\frac{1}{2}-i \xi, \quad q=-\frac{\gamma^{2}}{2}, \quad a=0 \\
b=\frac{1}{2}+\frac{i \beta \lambda}{2}, \quad c=\frac{\delta+1}{2} .
\end{gathered}
$$

The parameters of a Heun equation [13,14] are constrained, by the general theory of Fuschsian equations, as $\rho+\sigma+v=$ $a+b+1$, which provides us the first constraint relation for the chirping parameters $\zeta, \eta, \xi$ as

$$
\zeta+\eta+\xi=0
$$




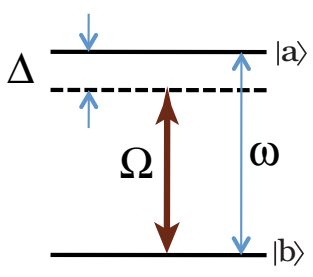

(a)

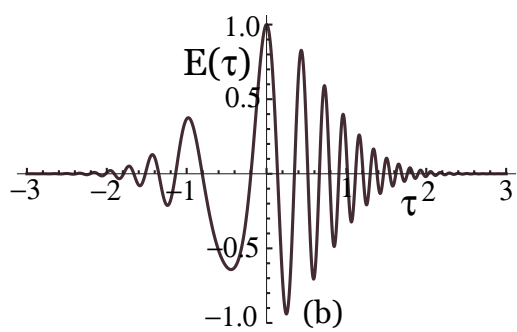

FIG. 1. (Color online) (a) Two-level atomic system, atomic transition frequency $\omega=\omega_{a}-\omega_{b}$, detuning $\Delta=\omega-v$, and Rabi frequency $\Omega(t)=\wp \mathcal{E}(t) / 2 \hbar$. (b) Classical electromagnetic field $E(t)=\exp \left(-\alpha^{2} t^{2}\right) \cos \left(v t+t^{2}\right)$.

The quantity $v+\dot{\phi}$ is the instantaneous pulse frequency; thus $\dot{\phi}$ should vanish for maximum $\Omega(\tau)$. From Eq. (5a), we get the corresponding $\varphi_{0}$, which satisfies the equation

$$
\lambda \varphi^{3}-(2 \lambda+\mu) \varphi^{2}+c(\lambda+2 \mu) \varphi-c \mu=0 .
$$

Thus the second constraint relation for the chirping parameters is given as

$$
\left(\frac{-2 c \zeta+2[(\zeta+\xi)+c(\zeta+\eta)] \varphi_{0}}{\left(\varphi_{0}-c\right)\left(\mu+\lambda \varphi_{0}\right)}\right)=0 .
$$

The general solution for Eq. (6), which has a regular singularity at $\varphi=0$, is given in terms of Heun local solutions $\mathcal{H}_{l}(\varphi)$ as

$$
\begin{aligned}
C_{a}= & \mathcal{P}_{1} \varphi^{1-\rho} \mathcal{H}_{l}[c, q+(1-\rho)\{(c-1) \sigma+a+b-\rho+1\} ; \\
& a-\rho+1, b-\rho+1,2-\rho, \sigma ; \varphi] \\
& +\mathcal{P}_{2} \mathcal{H}_{l}[c, q ; a, b, \rho, \sigma ; \varphi],
\end{aligned}
$$

where the constants $\mathcal{P}_{1}$ and $\mathcal{P}_{2}$ can be found using the initial conditions of the system. In the limit $\tau \rightarrow \infty$, the population left in the level $|a\rangle$ can be obtained by substituting $\varphi \rightarrow 1$ in Eq. (11). Let us consider the simple case of $\mu=1, \lambda=0$ in Eq. (4); then Eq. (5a) gives

$$
\begin{gathered}
\varphi(\tau)=\frac{1+\tanh (\tau)}{2}, \\
\Omega(\tau)=\gamma\left(\frac{2 \varphi(1-\varphi)}{(c-\varphi)}\right)^{1 / 2} .
\end{gathered}
$$

From Eq. (12), the pulse takes the form

$$
\Omega(\tau)=\frac{\gamma \operatorname{sech}(\tau)}{\sqrt{\delta-\tanh (\tau)}}, \quad \delta=2 c-1 .
$$

This pulse shape serves as an excellent model for a smooth box pulse, by taking care of nonanalyticity at its edges with the help of the pulse parameter $\delta$. Using Eq. (9), we get $\varphi_{0}=$ $c \pm \sqrt{c^{2}-c}$. From one of our earlier assumptions, $c>1$, only one of the possible values is allowed for $\varphi_{0}$ as $0 \leqslant \varphi_{0} \leqslant 1$. Subsequently using $\varphi_{0}=c-\sqrt{c^{2}-c}$ in Eq. (10), we get the constraint equation as

$$
\frac{\zeta-\eta}{\xi}=\delta-\sqrt{\delta^{2}-1} .
$$

The defining equation for the chirping function takes the form $\phi(\tau)=\xi\left\{\left(\delta-\sqrt{\delta^{2}-1}\right) \tau+\ln [\delta \cosh (\tau)-\sinh (\tau)]\right\}$.
For the pulse defined by Eq. (13), by use of the scaling parameters Eq. (2) and the chirping function Eq. (15), Eq. (3) gives

$$
\begin{aligned}
\ddot{C}_{a}(\tau) & -\left[\frac{1}{2}\left(\frac{1-2 \delta \tanh \tau+\tanh ^{2} \tau}{\delta-\tanh \tau}\right)\right. \\
& \left.-i \xi\left(\sqrt{\delta^{2}-1}-\frac{\delta^{2}-1}{\delta-\tanh (\tau)}\right)+i \beta\right] \dot{C}_{a}(\tau) \\
& +\frac{\gamma^{2} \operatorname{sech}^{2} \tau}{\delta-\tanh \tau} C_{a}(\tau)=0 .
\end{aligned}
$$

Let us define the initial conditions for our system as

$$
C_{a}(\tau \rightarrow-\infty)=0, \quad\left|C_{b}(\tau \rightarrow-\infty)\right|=1 .
$$

The solution for Eq. (16) satisfying the initial conditions is

$$
\begin{aligned}
C_{a}= & \mathcal{P} \varphi^{1-\rho} \mathcal{H}_{l}[c, q+(1-\rho)\{(c-1) \sigma+a \\
& +b-\rho+1\} ; a-\rho+1, b-\rho+1,2-\rho, \sigma ; \varphi],
\end{aligned}
$$

where $\varphi(\tau)$ is given by Eq. (12a) and the Heun parameters are

$$
\begin{gathered}
\rho=\frac{1}{2}-i\left(\zeta+\frac{\beta}{2}\right), \quad \sigma=\frac{1}{2}+i\left(\frac{\beta}{2}-\eta\right), \\
v=\frac{1}{2}-i \xi, \quad q=-\frac{\gamma^{2}}{2}, \quad a=0, \\
b=\frac{1}{2}, \quad c=\frac{\delta+1}{2} .
\end{gathered}
$$

The chirping parameters $\zeta, \eta$ and the constant $\mathcal{P}$ are given as

$$
\begin{gathered}
\zeta=-\frac{\xi}{2}\left(1-\delta+\sqrt{\delta^{2}-1}\right), \\
\eta=-\frac{\xi}{2}\left(1+\delta-\sqrt{\delta^{2}-1}\right), \\
\mathcal{P}=i \gamma\left(\frac{2^{(1-i \xi)}(1+\delta)^{i \xi-1 / 2}}{1+i(2 \zeta+\beta)}\right) .
\end{gathered}
$$

Here we have kept $\xi$ as a free parameter for the chirping function $\phi(t)$. In Fig. 2, we have considered some forms of the chirping function $\phi(\tau)$ [see Figs. 2(a) and 2(b)] for $\delta>1$ and $\delta \approx 1$, respectively, given by Eq. (15). The influence of chirping on the evolution of the probability amplitude for the upper level $|a\rangle$ in shown in Fig. 2(d) for the corresponding pulses in Fig. 2(c).

\section{B. Class II: Confluent Heun equation}

In this section, we consider another class of pulse and the corresponding chirping function. Let us define the pulse and the chirping function as

$$
\begin{aligned}
\Omega(\tau) & =\frac{2 \sqrt{2} \gamma(1-\varphi) \sqrt{\varphi}}{\mu+\lambda \varphi}, \\
\dot{\phi}(\tau) & =\frac{2 \zeta-2(\zeta+\eta) \varphi}{\mu+\lambda \varphi} .
\end{aligned}
$$

In terms of the variable $\varphi(\tau)$ and the definition of $\Omega(\tau)$ and $\dot{\phi}(\tau)$ from Eq. (5), Eq. (3) takes the form

$$
\frac{d^{2} C_{a}}{d \varphi^{2}}+\left(\frac{u}{\varphi}+\frac{v}{\varphi-1}\right) \frac{d C_{a}}{d \varphi}+\frac{(p \varphi+q) C_{a}}{\varphi(\varphi-1)}=0,
$$



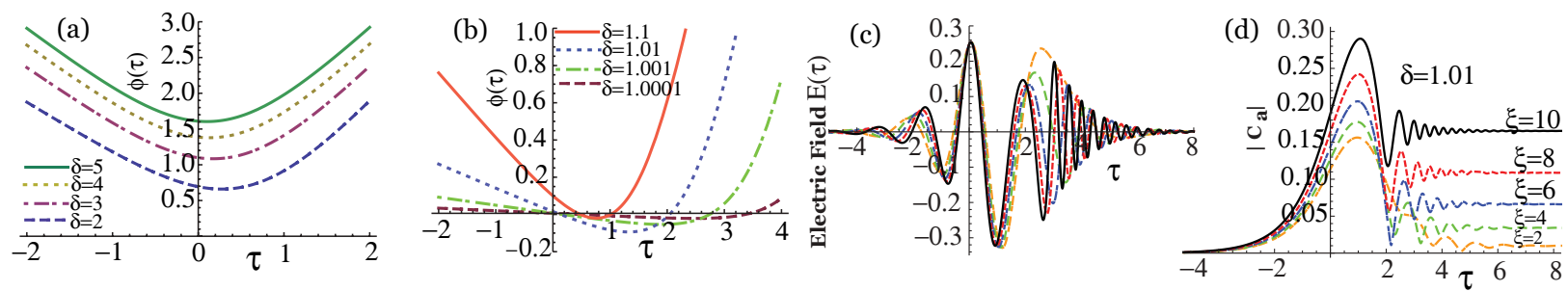

FIG. 2. (Color online) (I) Heun equation case: Chirping function $\phi(\tau)$ given by Eq. (15) for $\xi=10$ and (a) $\delta>1$ and (b) $\delta \approx 1$. (c) Electric field $E(\tau)$ for varying $\xi$ and $\delta=1.01$. (d) Probability amplitudes for the upper level $|a\rangle$ for the corresponding fields in (c). $\left|C_{a}(\tau)\right|$ is given by Eq. (18).

where

$$
\begin{gathered}
u=\frac{1}{2}-i\left(\zeta+\frac{\beta \mu}{2}\right), \quad v=i\left(\frac{\beta(\lambda+\mu)}{2}-\eta\right), \\
p=-q=2 \gamma^{2} .
\end{gathered}
$$

The critical point, which corresponds to the peak of $\Omega(\tau)$, is given by

$$
\varphi_{0}=-\left(\frac{\lambda+3 \mu-\sqrt{(\lambda+\mu)(\lambda+9 \mu)}}{2 \lambda}\right),
$$

and the corresponding $\tau_{0}$ can be found using Eq. (4). At this point $\dot{\phi}=0$, which gives a constraint relation as

$$
\begin{aligned}
& 3 \zeta(\lambda+\mu)-\zeta \sqrt{(\lambda+\mu)(\lambda+9 \mu)} \\
& \quad+\eta(\lambda+3 \mu-\sqrt{(\lambda+\mu)(\lambda+9 \mu)})=0 .
\end{aligned}
$$

The general solution of the confluent Heun equation (22) is given as

$$
\begin{aligned}
C_{a}= & \mathcal{P}_{1} \mathcal{H}_{l}^{(c)}[0, u-1, v-1, p, q+(1-u v) / 2, \varphi] \\
& +\mathcal{P}_{2} \varphi^{1-u} \mathcal{H}_{l}^{(c)}[0,1-u, v-1, p, q+(1-u v) / 2, \varphi],
\end{aligned}
$$

where $\mathcal{P}_{1}$ and $\mathcal{P}_{2}$ can be found using the initial condition of the system. In the limit $\tau \rightarrow \infty$, the population left in the level $|a\rangle$ can be obtained by substituting $\varphi \rightarrow 1$ in Eq. (26). Let us consider the simple case of $\mu=1, \lambda=0$. Thus the new variable is given by Eq. (12a). From Eqs. (24) and (25) we get $\varphi_{0}=1 / 3$ and $\eta=2 \zeta$. The pulse shape $\Omega(\tau)$ and the chirping function can be written as

$$
\begin{gathered}
\Omega(\tau)=\gamma \operatorname{sech}(\tau)[1-\tanh (\tau)]^{1 / 2}, \\
\dot{\phi}(\tau)=-\zeta[1+3 \tanh (\tau)] .
\end{gathered}
$$

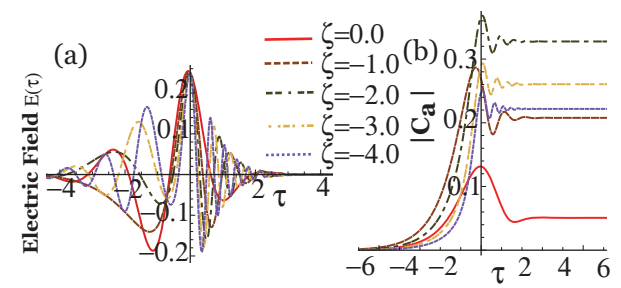

FIG. 3. (Color online) (II) Confluent Heun equation case: (a) Profile of the electric field $E(\tau)$ for varying $\zeta$. (b) Probability amplitudes for the upper level $|a\rangle$ for the corresponding fields in (a). The pulse envelope $\Omega(\tau)$ and are given by Eq. (27).
For the pulse defined by Eq. (27a), by use of the scaling parameters Eq. (2) and the chirping function Eq. (27b), Eq. (3) gives

$$
\begin{gathered}
\ddot{C}_{a}(\tau)-\left(i \beta-\frac{1}{2}[1+3 \tanh (\tau)]-i \zeta[1+3 \tanh (\tau)]\right) \dot{C}_{a}(\tau) \\
+\gamma^{2} \operatorname{sech}^{2}(\tau)[1-\tanh (\tau)] C_{a}(\tau)=0 .
\end{gathered}
$$

Let us define the initial conditions for our system as Eq. (17). The solution for Eq. (28) satisfying the initial conditions is

$C_{a}=\mathcal{P} \varphi^{1-u} \mathcal{H}_{l}^{(c)}[0,1-u, v-1, p, q+(1-u v) / 2, \varphi]$,

where $\varphi(\tau)$ is given by Eq. (12a) and the Heun parameters are $u=\frac{1}{2}-i\left(\zeta+\frac{\beta}{2}\right), \quad v=i\left(\frac{\beta}{2}-\eta\right), \quad p=-q=2 \gamma^{2}$.

The constant $\mathcal{P}$ is given as

$$
\mathcal{P}=\gamma\left(\frac{2^{(3 / 2+3 i \zeta)}}{(2 \zeta+\beta)-i}\right)
$$

Here we have kept $\zeta$ as a free parameter for the chirping function $\phi(\tau)$ given by Eq. (27). The influence of chirping

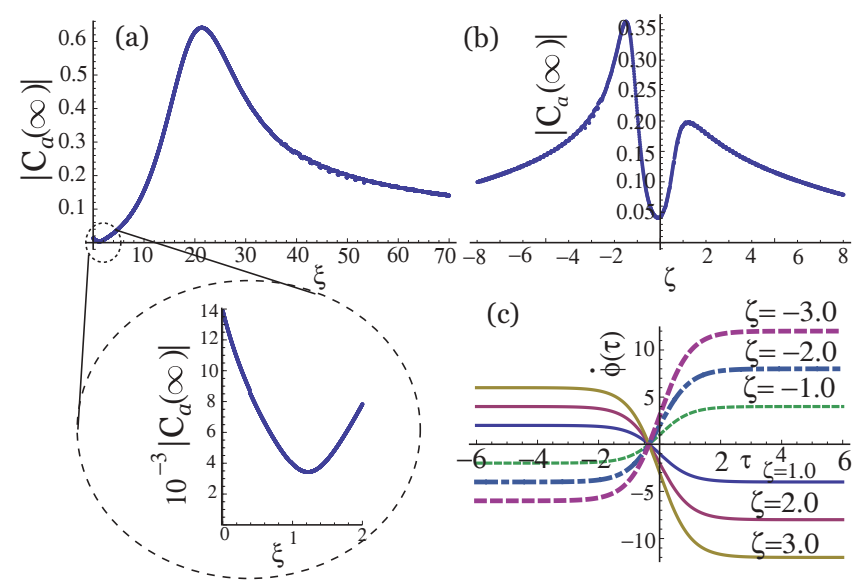

FIG. 4. (Color online) Effect of chirping on the population left in the excited states $|a\rangle$. (a) Heun equation and (b) confluent Heun equation. The inset shows the dip (minima) in the population left for the Heun case. For the calculations, $\beta=2.5, \gamma=0.25$, and $\delta=1.01$. (c) Chirping function for the confluent Heun case, $\dot{\phi}(\tau)$. 

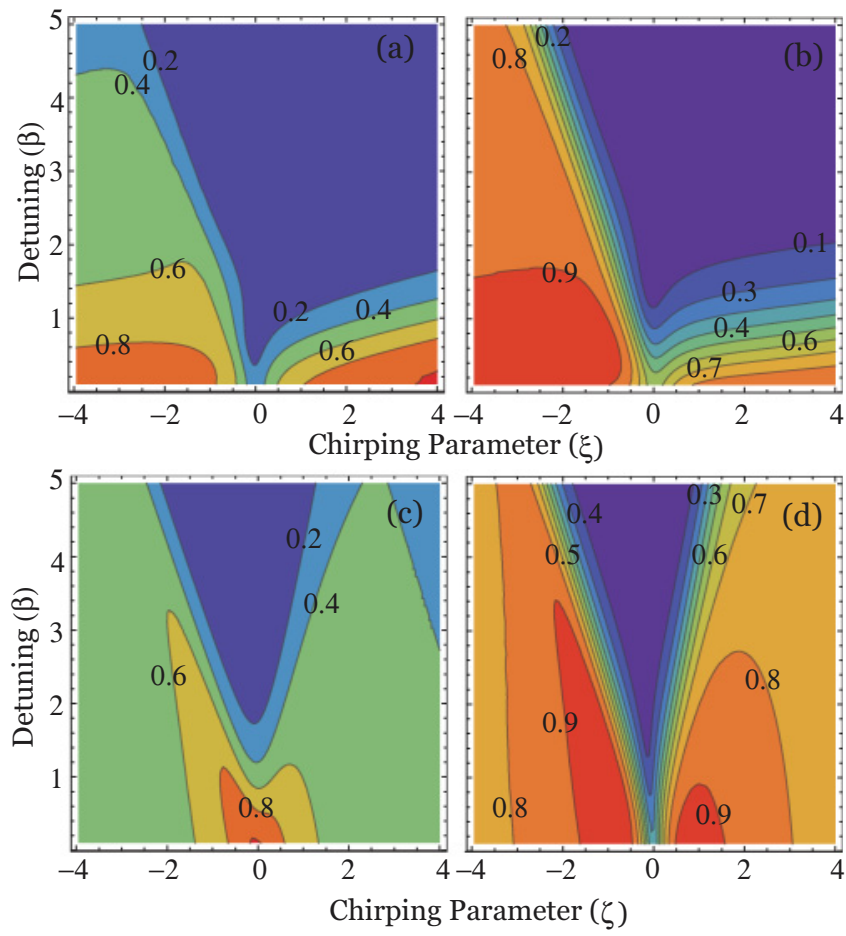

FIG. 5. (Color online) Contour plot showing the effect of chirping and the detuning on the population left in the excited states $|a\rangle$. (a) and (b) correspond to the Heun and (c) and (d) to the confluent Heun case. For calculations, (a) $\gamma=0.50, \delta=1.01$, (b) $\gamma=1, \delta=$ 1.01 , (c) $\gamma=0.5$, and (d) $\gamma=1$.

on the evolution of the probability amplitude for the upper level $|a\rangle$ in shown in Fig. 3(b) for the corresponding pulses in
Fig. 3(a). To see the effect of chirping on the population left in the upper level $|a\rangle$, we have plotted in Fig. $4\left|C_{a}(\infty)\right|$ as a function of the free chirping parameter for the Heun and the confluent Heun cases for a particular choice of the detuning $\beta$ and the peak Rabi frequency $\gamma$. We see that $\left|C_{a}(\infty)\right|$ ranges from $4 \times 10^{-3}$ to $\backsim 6 \times 10^{-1}$. In Fig. 5 we have plotted the population left in the upper level $|a\rangle$ as a function of detuning $\beta$ and the free chirping parameters $\xi$ and $\zeta$ for the Heun and the confluent Heun cases, respectively.

In conclusion, we have found solutions for the problem of a two-level system driven by chirped pulses in exact analytical form. The solutions are given in terms of the Heun function $\mathcal{H}_{l}$ and the confluent Heun function $\mathcal{H}_{l}^{(c)}$. Using the appropriate chirping parameters, we have shown that the population left after the pulse is gone can be enhanced by four orders of magnitude [see Fig. 4(a) inset]. The application of chirped pulses to the population transfer or generation of coherence makes it very interesting to look for other shapes for which the system can be solved analytically, because finding exact analytical solutions for such a problem will not only supplement numerical simulations but will also be useful in understanding the underlying physics.

We thank M. O. Scully, L. Keldysh, and M. S. Zubairy for useful discussions and gratefully acknowledge the support from the NSF Grant No. EEC-0540832 (MIRTHE ERC), the Office of Naval Research (Grants No. N00014-09-10888 and No. N0001408-1-0948), the Robert A. Welch Foundation (Award No. A-1261) and partial support from the CRDF. P.K.J would also like to acknowledge the Robert A. Welch Foundation and HEEP Foundation for financial support.
[1] Claude Cohen-Tannoudji, Atom-Photon Interactions: Basic Processes and Applications (New York, Wiley, 1992).

[2] M. O. Scully et al., Proc. Natl. Acad. Sci. U.S.A. 99, 10994 (2002).

[3] M. O. Scully, Y. Rostovtsev, A. Svidzinsky, and Jun-Tao Chang, J. Mod. Opt. 55, 3219 (2008).

[4] P. Jaegle, Coherent Sources of XUV Radiation (Springer, New York, 2005).

[5] A. W. Weiner, Rev. Sci. Instrum. 71, 1929 (2000).

[6] M. Shapiro and P. Brumer, Principles of the Quantum Control of Molecular Processes (Wiley, Hoboken, NJ, 2003); T. Brixner and G. Gerber, Chem. Phys. Chem. 4, 418 (2003); H. Rabitz, M. Motzkus, K. Kompa, and R. de Vivie-Riedle, Science 288, 824 (2000).

[7] Y. V. Rostovtsev, H. Eleuch, A. Svidzinsky, H. Li, V. Sautenkov, and M. O. Scully, Phys. Rev. A 79, 063833 (2009).

[8] P. K. Jha and Y. V. Rostovtsev, Phys. Rev. A 81, 033827 (2010).
[9] M. O. Scully and M. S. Zubairy, Quantum Optics (Cambridge University Press, Cambridge, England, 1997).

[10] L. Allen and J. H. Eberly, Optical Resonance and TwoLevel Atoms (Cambridge University Press, Cambridge, England, 1997).

[11] Here we use the convention that all frequencies are circular frequencies, so that $\hbar v($ not $h v$ ) is the photon energy.

[12] F. T. Hioe, Phys. Rev. A 30, 2100 (1984); F. T. Hioe and C. E. Carroll, ibid. 32, 1541 (1985); J. Zakrzewski, ibid. 32, 3748 (1985); C. E. Carroll and F. T. Hioe, ibid. 41, 2835 (1990); D. Goswami and W. S. Warren, ibid. 50, 5190 (1994); E. J. Robinson, J. Phys. B 18, 3687 (1985); P. A. Ivanov and N. V. Vitanov, Phys. Rev. A 71, 063407 (2005); A. Ishkhanyan and K.-A. Suominen, J. Phys. A 34, 6301 (2001).

[13] A. Ronveaux, Heun's Differential Equations (Oxford University Press, Oxford, 1995).

[14] K. Heun, Math. Ann. 33, 161 (1889); N. Gurappa and P. K. Panigrahi, J. Phys. A Math. Gen. 37, L605 (2004); R. S. Maier, Math. Comp. 76, 811 (2007). 